\title{
Exploration of Attendance, Active Participation, and Behavior Change in a Group-Based Responsive Stimulation, Maternal and Child Health, and Nutrition Intervention
}

\author{
Farzana Yeasmin, ${ }^{1 \star}$ Peter J. Winch, ${ }^{2}$ Sharon T. Hwang, ${ }^{2}$ Elli Leontsini, ${ }^{2}$ Tania Jahir, ${ }_{1}^{1}$ Jyoti B. Das, ${ }^{1}$ Mohammad R. Amin, ${ }^{1}$ \\ Md K. Hossain, ${ }^{1}$ Tarique Md. Nurul Huda, ${ }^{1}$ Fahmida Akter, ${ }^{1}$ Abul Kashem Shoab, ${ }^{1}$ Fahmida Tofail, ${ }^{1}$ Malay K. Mridha, ${ }^{3}$ \\ Jesmin Sultana, ${ }^{1}$ Helen Pitchik, ${ }^{4}$ Lia C. H. Fernald, ${ }^{4}$ Stephen P. Luby, ${ }^{5}$ and Mahbubur Rahman ${ }^{1}$ \\ ${ }^{1}$ Environmental Interventions Unit, Infectious Disease Division, icddr,b, Dhaka, Bangladesh; ${ }^{2}$ Johns Hopkins Bloomberg School of Public Health, \\ Baltimore, Maryland; ${ }^{3}$ James P. Grant School of Public Health, Dhaka, Bangladesh; ${ }^{4}$ School of Public Health, University of California, Berkeley,
} Berkeley, California; ${ }^{5}$ Stanford University, Stanford, California

\begin{abstract}
Delivery of interventions through group sessions allows for in-depth discussions and creates opportunities for group members to work together to identify and solve common problems. However, low attendance may limit impact. We explored factors affecting attendance, active participation, and behavior change in an integrated group-based child development and maternal and child health intervention in Bangladesh. Community health workers (CHWs) facilitated two sessions a month including material on child stimulation; water, sanitation, and hygiene; nutrition, maternal depression, and lead exposure prevention. Sessions were conducted with 320 pregnant women and mothers with children younger than 24 months, in 16 villages in Kishoreganj district. After 4 and 9 months of intervention, we conducted focus group discussions and in-depth interviews with mothers $(n=55$ and $n=48)$ to identify determinants of attendance and behavior change, and to examine potential for intervention scale-up. Recruiting family members to assist with childcare resulted in improved attention during sessions. Adopting a storytelling format for presentation of session materials resulted in more engaged participation during courtyard sessions. Session attendance and behavior change, especially purchasing decisions, were difficult for participants without the support of male heads of the household. Selecting a rotating leader from among the group members to remind group members to attend sessions and support CHWs in organizing the sessions was not successful. Facilitating self-appraisals and planning for water and sanitation allowed participants to identify areas for improvement and track their progress. Key determinants of a participant's attendance were identified, and the resulting intervention shows promise for future implementation at scale.
\end{abstract}

\section{INTRODUCTION}

Child health, growth, and development in low- and middle-income countries (LMICs). Millions of children younger than 5 years in LMICs face a multitude of risks in their environment which impact their physical and cognitive development. ${ }^{1}$ An estimated $22 \%$ of children are born with low birth weight, ${ }^{2}$ and 155 million children younger than 5 years were affected by stunting in 2016, most living in Asia and Africa. ${ }^{3}$ Many of these children grow up in environments heavily contaminated with fecal pathogens, exposed to environmental lead, ${ }^{4,5}$ and experience limited parent-child interaction and stimulation activities, which may result in poor cognitive development and impairment in intellectual function. ${ }^{6}$

Several focused interventions improve early child development. Interventions encouraging mothers and adult family members to practice responsive stimulation with their children improve child cognitive development. ${ }^{7,8}$ Micronutrient deficiencies are a powerful individual-level predictor of child development outcomes beyond height and weight, ${ }^{9}$ and multiple studies have demonstrated that provision of multiple micronutrient supplements to pregnant women improves child birth weight ${ }^{10}$ and child cognition through 2 years of age. ${ }^{11}$ Interventions to improve infant and young child nutrition, specifically breastfeeding and appropriate complementary feeding interventions, have significant beneficial effects on child development. $^{12,13}$ Maternal mental health is important for

${ }^{*}$ Address correspondence to Farzana Yeasmin, Environmental Interventions Unit, Infectious Disease Division, icddr,b, 68 Shahid Tajuddin Ahmed Sharani, Dhaka, 1212, Bangladesh. E-mail: fyeasmin@icddrb.org children younger than 3 years who are highly dependent on mothers for best nutrition and stimulation practices. ${ }^{14}$

Attendance and participation in integrated group sessions for child development. With the ultimate goal to improve early child development in rural Bangladesh, we aimed to develop integrated child stimulation, water, sanitation, and hygiene (WASH); maternal and child nutrition; lead exposure prevention; and maternal mental health intervention. We chose to focus on group sessions because they 1) allow for indepth discussion of difficult topics such as maternal depression; 2) build solidarity among group members ${ }^{15-17}$; 3) create a forum for sharing of experiences, allowing group members to work together to identify and solve common problems; and 4) there is some evidence for impact from group-based child stimulation interventions on child development in prior studies, ${ }^{18}$ and they may have lower cost than individual household visits.

Despite documented success, operational challenges commonly occur during group session implementation, such as poor attendance. ${ }^{19}$ Exploring determinants for attendance at individual sessions can help improve delivery and effectiveness of group sessions. ${ }^{20}$ Attendance at group sessions depends on a variety of factors, and several strategies have been previously documented to support higher attendance rates. In one study, reasons for nonattendance included scheduling the session at an inconvenient time for the participants and holding sessions at difficult to reach locations. ${ }^{21}$ In another study, family stress hindered group session attendance. ${ }^{19}$ Economic, educational, social, and health barriers can also cause low attendance, and consequently, refreshments, transportation, and childcare provided to disadvantaged families helped them overcome the economic barrier to attending sessions. ${ }^{21}$ Incentives including gifts, transportation, 
free food, childcare, and monetary rewards have previously been used as strategies to encourage parents to attend group sessions, although these can prove costly and may not be sustainable in low-resource settings. ${ }^{22}$

In addition to addressing barriers to session attendance, it is important to identify barriers to adoption of recommended behaviors for participants who do attend the group sessions. One potential barrier is promotion of many behavioral recommendations at the same time in a resource-constrained setting. ${ }^{23}$ An additional barrier can be the absence of household decision-makers during the sessions. Even if the session attendee knows how to change behavior, they may lack the necessary decision-making autonomy to bring about change if it involves changes in purchasing behavior or behaviors that require input from others in the household. ${ }^{24}$ Involving household decision-makers to identify and address barriers to adopting behavioral recommendations is important for such an intervention.

Engaging participants actively in sessions may promote attendance as well as the adoption of recommended behaviors presented in these sessions. Group sessions may be more engaging for the participants. ${ }^{25}$ There are several intervention models incorporating group sessions. These sessions require a larger time commitment by participants than individual sessions conducted in the home, but may result in improved outcomes. Group antenatal care (GANC) is one of the intervention models. A study on GANC in Senegal found that group participation may enhance opportunities to gain knowledge, experience, and necessary skills for improved childbirth because of its focus on relationships and nurturing practices. $^{26}$ As such, this may lead to developing transformational relationships among women and their families. ${ }^{27}$ Another model is participatory women's groups for maternal and newborn health, actively facilitated by local women. In a study in Nepal, women who participated in sessions of these women's groups were able to successfully develop various strategies to reduce barriers of maternal and newborn care. ${ }^{28}$ This participatory intervention demonstrated that local communities were capable of establishing women's groups facilitated by paid local women to identify problems and brainstorm community planning strategies to tackle these problems. ${ }^{28}$ Reducing barriers to attend sessions during the initial stage of the intervention implementation can increase active participation. ${ }^{29}$ We do not have similar evidence for the integrated intervention model discussed in this article.

The Research on Integration of maternal and child Nutrition, Early childhood development, and WASH (RINEW) study team developed an integrated early child development intervention delivered through group sessions with pregnant women and mothers of young children. The intervention included components on child stimulation, maternal and child nutrition, maternal depression, WASH, and prevention of lead exposure to improve child development in a resource-limited context. One goal of the study was to develop strategies to mitigate the barriers to implementation that could be applied at a larger scale. The objective of this study was to conduct qualitative research to explore factors affecting attendance, active participation, and adoption of behavioral recommendations in the RINEW group-based intervention package.

\section{METHODS}

Methods of intervention. The integrated RINEW intervention was piloted in 320 households in 16 villages of the Katiadi and Kuliarchar subdistricts of Kishoreganj district. Each village held a total of 18 sessions, two sessions per month, facilitated by a community health worker (CHW). Community health workers were community members with nine or more years of education, trained by the study team to deliver the intervention. Before the start of the intervention, $\mathrm{CHW}$ s enrolled in 8 days of basic training followed by 4 days of refresher training, including training on tablet application. Over the course of the intervention, $\mathrm{CHW}$ s received nine additional trainings for the upcoming sessions, each of which took 23 days. The training included didactic sessions, in-class practice, and field practice. In eight villages, $\mathrm{CHWs}$ held 18 group sessions with neighboring women who were either pregnant or mothers of children younger than 24 months (group arm), and in eight other villages, CHWs alternated group sessions and home visits (combined arm) so that each household received both a group session and a home visit each month (Table 1).

Participants and their children were invited to the intervention sessions. All enrolled pregnant women and mothers with children younger than 6 months were provided multiple micronutrients called "Solvit-M." Arm circumference was used to indicate nutritional status and thus vulnerability to other early child risk factors. ${ }^{30,31}$ Households of children whose middle-upper arm circumference measurement was between $11.5 \mathrm{~cm}$ and $<12.5 \mathrm{~cm}$ were selected as targeted households to receive additional inputs including micronutrients with lipid-based nutrient supplements for children older than 6 months, pit latrines, child potties, and handwashing stations. Children older than 6 months with a middleupper arm circumference of 12.5 and above were provided with micronutrient powders called "Pushtikona." In addition, all the breastfeeding mothers were provided age-specific picture books, a soapy water bottle, and detergent packets.

Intervention sessions were designed to be participatory and were facilitated with pictorial cards, and included group-

TABLE 1

Phase I and phase II: description of participants' involvement in the qualitative assessments

\begin{tabular}{|c|c|c|c|c|c|}
\hline & & & & & \\
\hline & & $\begin{array}{c}\text { No. of } \\
\text { focus groups }\end{array}$ & $\begin{array}{l}\text { No. of in-depth } \\
\text { interviews }\end{array}$ & $\begin{array}{c}\text { No. of } \\
\text { focus groups }\end{array}$ & $\begin{array}{l}\text { No. of in-depth } \\
\text { interviews }\end{array}$ \\
\hline Phase I. First qualitative & High-attendance mothers & 2 & 5 & 2 & 5 \\
\hline assessment $(n=59)$ & Low-attendance mothers & 2 & 1 & 1 & 1 \\
\hline Phase II. Second qualitative & High-attendance mothers & 2 & 0 & 2 & 0 \\
\hline assessment $(n=44)$ & Males & 0 & 2 & 0 & 2 \\
\hline & Low-attendance mothers & 0 & 3 & 0 & 5 \\
\hline & Targeted mothers & 0 & 5 & 0 & 4 \\
\hline
\end{tabular}


based household environmental assessments and planning, and coaching of caregiver-child interactions with agespecific toys and picture books. Pictorial cards were used to educate mothers on of the 10 food groups and further assisted in engaging mothers to actively participate in the sessions. These cards, with the support of CHWs, also taught mothers how to detect symptoms for common physiological changes during pregnancy and lactation, and their possible courses of action. The main behavioral recommendations delivered in each of the intervention components are shown in Table 2.

The intervention was designed based on previous formative research on four individual components, including psychosocial stimulation for early childhood development, ${ }^{32}$ WASH, maternal and child nutrition ${ }^{33-35}$ adapted thinking healthy, ${ }^{36,25}$ and lead exposure prevention. $^{5}$

Methods for qualitative process evaluation. Study design. We conducted two phases of qualitative assessments (Table 1) to inform intervention delivery and to explore the feasibility, acceptability, and scalability of the RINEW intervention package (Table 2). Phase 1 involved in-depth interviews and focus group discussions during month 4 of the intervention to understand mothers' challenges in attending the twice-monthly sessions and understanding and practicing the recommended behaviors. Phase 2 involved focus group discussions with mothers and in-depth interviews with both male heads of households and mothers following 9 months of intervention delivery to assess perceptions of the modified intervention package and delivery, based on the findings of phase 1.

Qualitative assessments were conducted by four experienced and trained anthropologists. All individual interviews lasted less than 60 minutes, and all the focus group discussions lasted less than 90 minutes. Interview and focus group guides were developed according to the objectives and corresponding research questions for each category of participants. For questions related to WASH, we developed questions corresponding to the dimensions and the aggregate levels in the Integrated Behavioral Model for Water Sanitation and Hygiene (IBM-WASH) model, ${ }^{37}$ an ecological model on WASH behavior change. To inform development of questions on motivation and performance of CHWs, we drew from Greenspan et al. ${ }^{38}$ and Rahman et al. ${ }^{39}$ specially Figure 1 "Framework for decision-making process in retention and attrition of CHWs" in Rahman et al. ${ }^{39}$

The data collection team used the questions in each module of the guides as the starting point in asking questions to the participants. For the in-depth interviews, a single interviewer conducted the interview. For the focus group discussions, two-three data collection team members were involved in facilitation, note taking, and ensuring the comfort of participants. These are examples of questions included in the guides for the semi-structured interviews:

1. Have you shared any of the messages that you learned from the sessions with any of your neighbors or relatives? Which ones?

TABLE 2

Pilot intervention content on child stimulation; maternal and child health and nutrition; water, sanitation, and hygiene (WASH); maternal mental health; and prevention of lead exposure

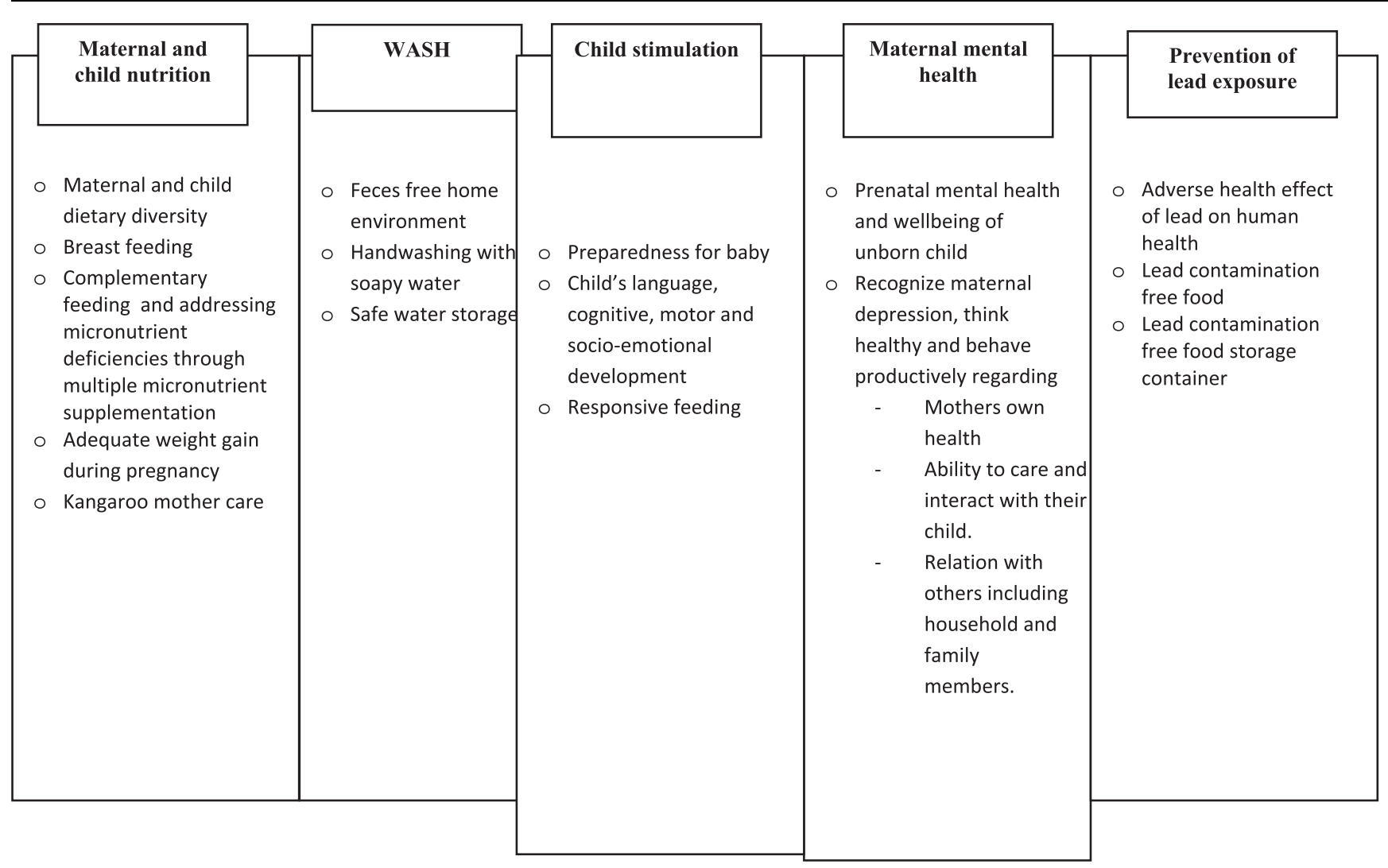


TABLE 3

Barriers and challenges in evaluating mothers' attendance at group sessions and practicing behavioral recommendations from group sessions Barriers and challenges Theme 1: Mothers and pregnant women
faced challenges in attending group
sessions

Theme 2: Mothers and pregnant women faced challenges during group sessions

Theme 3: Mothers and pregnant women faced challenges in practicing recommended behaviors presented during the group sessions
- Mothers move to their parents' house to deliver a child, which is sometimes far from their home

- General concern of participant or her child becoming sick during the winter months

- Male household members had the decision-making power to make the decision for the mothers to attend sessions

- Sickness of mothers and child's sickness

- Mothers move to and from their parents' house to visit

- Conflicting time commitments necessary for household-related tasks or harvesting with no assistance

- Disruption from children

- Difficulty concentrating for long periods of time, especially during longer sessions

- Unengaging material during the sessions

- Male household members hold purchasing power for sanitation hardware, and diverse nutritious foods. Mothers and pregnant women are often unable to invest without consent of their husbands

- Difficultly in washing hands after each of the recommended key times, as mothers are busy with household chores or caring for their child
2. If you were asked to share one activity or message with a mother who had not participated in the program, which activity or message would you share? Why?

3. Do you have any advice on how the program can be improved for other parents who participate at a later date?

4. What benefits did you get by practicing these recommended behaviors/activities? (Probe for each intervention component, e.g., preparing and using soapy water bottle, washing hands with soap on several key times, latrine improvement, food storage, and lead protection)

5. Have you noticed any changes in you and your child? What are the changes?

First qualitative assessment. We conducted seven focus group discussions (at least six participants in each) with 43 mothers to identify the challenges to attending and participating in group sessions and home visits (Table 1). Participants were stratified by group and combined arm, and high and low intervention exposure, based on the number of sessions attended. High exposure was categorized as having attended most of the sessions (80\%), and low-exposure participants attended few or no sessions (20\% or less). We stratified on exposure to understand the barriers and facilitators to regularly attending and participating in the intervention sessions. We assessed the understanding, acceptability, and feasibility of the behavioral recommendations promoted in the integrated package and participant's barriers to attending group sessions (Table 3). In the combined arm, we additionally identified the benefits and challenges to attending and participating in group sessions compared with home visits.

We conducted in-depth interviews with 12 mothers (Table 1) to assess the same factors for each of the components in further detail. In each intervention arm, five interviews were conducted with high-exposure mothers and one interview for low-exposure mothers (Table 1). We refined the intervention based on the findings of the first qualitative assessment.

Second qualitative assessment. We conducted a second qualitative assessment in the final month (month 9) of the intervention. We assessed participants' experiences, barriers, and facilitators in adopting behavioral recommendations and attending group sessions and home visits. We conducted focus groups with 27 mothers and individually interviewed 17 mothers and four fathers or grandfathers to gain further details for each intervention component (Table 1). Questions in the second phase centered on asking about the modifications that had been implemented: 1) participants' experience of the intervention content presented in a storytelling format, 2) participants' experience bringing family members for childcare assistance during the session, 3) participants' perception of incorporating household decision-makers into group discussions, 4) participants' perception of the new household environmental assessment and planning component, and 5) participants' experiences serving as a group leader and supporting CHWs' session organization.

Qualitative data analysis. All interviews and group discussions were conducted in Bengali (Bangla), the national language of Bangladesh. We recorded all data and renamed each according to the type of participant and the type of tools applied, and de-identified the data. We conducted manual coding of the data. This qualitative sub-study, being part of a larger impact study, was conducted under an objectivist epistemology. We followed the definition and dimensions of trustworthiness described in Krefting. ${ }^{40}$ The strategies that we followed to establish trustworthiness included prolonged and varied field experience by the qualitative interviewers, sampling at different points during implementation of the intervention (month 4 and 9), member checking by sharing analysis results with $\mathrm{CHWs}$, and triangulation of qualitative findings from interviews, focus groups, and various types of participants (pregnant women, mothers, and household decision-makers). We practiced reflexivity during meetings of the qualitative team with the study investigators, reflecting on the lens that the interviewers and analysts brought to their work, based on their social class, education and training, and professional identities.

Focus group discussions and in-depth interviews were documented with an audio recorder and handwritten notes, and transcribed verbatim from Bengali to English, paying special attention to local terms and expressions. In addition, handwritten field notes from informal discussions and observations were taken by the research team, as a way to record 
TABLE 4

Average duration of session and attendance to date of the integrated Research on Integration of maternal and child Nutrition, Early childhood development and WASH curriculum

\begin{tabular}{|c|c|c|c|c|}
\hline \multirow[b]{2}{*}{ Session } & \multicolumn{2}{|c|}{ Group-only arm } & \multicolumn{2}{|c|}{ Combined arm ${ }^{\star}$} \\
\hline & Duration $($ mean $\pm S D)$ & Attendance, $n(\%)$ & Duration $($ mean $\pm S D)$ & Attendance, $n(\%)$ \\
\hline One & $45 \pm 9.0$ & 142 (89) & $44 \pm 12$ & $143(90)$ \\
\hline Two & $39 \pm 13$ & 134 (84) & $33 \pm 14$ & $145(92)$ \\
\hline Three & $43 \pm 12$ & $134(84)$ & $40 \pm 12$ & $129(82)$ \\
\hline Four & $39 \pm 13$ & $132(84)$ & $35 \pm 15$ & 138 (88) \\
\hline Five & $39 \pm 11$ & $123(78)$ & $32 \pm 13$ & 131 (83) \\
\hline Six & $33 \pm 12$ & $124(79)$ & $34 \pm 14$ & $131(83)$ \\
\hline Seven & $39 \pm 15$ & $120(76)$ & $34 \pm 16$ & $127(81)$ \\
\hline Eight & $38 \pm 11$ & $117(75)$ & $31 \pm 16$ & $143(91)$ \\
\hline Nine & $31 \pm 13$ & $115(73)$ & $33 \pm 11$ & $130(83)$ \\
\hline Ten & $37 \pm 14$ & $123(78)$ & $35 \pm 16$ & 132 (84) \\
\hline Eleven & $35 \pm 13$ & $112(71)$ & $35 \pm 13$ & $123(78)$ \\
\hline Twelve & $32 \pm 13$ & $117(75)$ & $32 \pm 16$ & 135 (86) \\
\hline
\end{tabular}

unspoken yet critical observations from the sessions. ${ }^{41}$ The tone and attitude of respondents was noted. These notes were included in the thematic analysis.

The research team met regularly during the data transcription and translation process to discuss the best way to convey the meaning in English. Most codes were generated before analysis, based on the same guiding models used to develop the field guides, namely, the IBM-WASH model, ${ }^{37}$ Rahman et al., ${ }^{39}$ and Greenspan et al. ${ }^{38}$ As coding proceeded, additional inductive codes were defined and applied when new themes not covered by our guiding models emerged. The codes encompassed barriers and facilitators for each intervention component and delivery modality. Data were analyzed first using a debriefing format in which the research team conducted an initial analysis by reading through transcripts and field notes taken by interviewers, ${ }^{42}$ followed by a more systematic thematic analysis using the deductive codes that were generated before data collection, as well as any inductive codes generated during data analysis.

Interviewers obtained both verbal and written consent from all study participants. The study protocol was reviewed and approved by the Ethical Review Committee of icddr, $b$ and by the Committee for the Protection of Human Subjects at the University of California, Berkeley.

\section{RESULTS}

Group session attendance by mothers. The participants' attendance was recorded on tablets at the start of each session. We observed varied session attendance in both the group and combined arms as shown in Table 4. Attendance was more than $80 \%$ until session four and more than $70 \%$ for the remainder of the sessions.

Throughout the intervention period, combined arm participants attended on average one more session (group attendance $=14.8$, mixed $=13.8$ ), and this difference was due to attending a higher number of home visits.

First phase of qualitative assessment. Interviews and focus groups in the first phase were designed to understand the acceptability and feasibility of the integrated intervention package and participant's barriers to attending group sessions. We imposed the category of "barriers" during the analysis. During the data collection, we were trying to understand the sequence of events that resulted in women attending and participating in the group sessions, or not. Women did not describe or connect these events with any one term, but different women in different interviews did use Bengali-language terms such as jhamela or shomosha that correspond to the English words difficulties or barriers. We represent these different Bengali-language terms with the English word barriers, but this English word includes a wide range of experiences and concerns, that we report in this manuscript with quotes from the women.

Barriers to attendance. Mothers had difficulty in regularly attending group sessions. The barriers included the cultural practice that pregnant woman move into her parents' home during the delivery and postpartum period, ${ }^{40}$ reluctance to take children outside during winter due to concerns of getting sick, mother's own sickness, and ongoing pressure to complete expected duties such as household chores or harvesting. A 30-year-old mother from combined arm said,

\section{If the meeting is held during harvesting time then it is very problematic for me to concentrate in the session}

Most mothers reported that husbands generally did not help them with household chores as housework continues to be seen as the primary responsibility of the women within the household.

Barriers to paying attention. Some mothers who attended group sessions faced difficulty managing active young children during the sessions, impacting their ability to pay attention to session content. A 27-year-old mother from combined arm stated,

My baby defecated in a session, I had to clean her, and I had to leave the session for a while, and I missed some discussion. When I came back I could not pay attention. Sometimes my baby cries and annoys me, I had to pacify her, and I got distracted from the discussion.

The long duration of sessions, lasting generally from 45 minutes to more than 1 hour, which were held in the courtyards 
of the participants' households, proved difficult to maintain some of the mothers' continued attention. Some mothers stated the session duration made it hard to pay attention.

Experiences with content. Many mothers were eager to hear from CHWs about new topics and could identify the benefits of the session's contents. Twenty-one mothers stated they were likely to share new concepts learned during the sessions while interacting with other mothers, family members, and their children. When asked about topics covered in the sessions that they might share or discuss with others, mothers most commonly $(N=10)$ volunteered that they would share information about lead and its negative impact. Their enthusiasm for sharing lessons learned stemmed from their newfound knowledge on how they could facilitate preventing adverse effects on human health especially and children's cognitive development.

However, several mothers were not eager or did not share content that they had learned in the group. Six mothers had not shared any content with their household members, and only one had shared content with their household members related to playing with children. Primary reasons for this included preference in discussing and receiving health-related messages solely from health providers and lack of time in discussing lessons learned with others.

Barriers to practicing behavioral recommendations. A number of women reported finding the session content to be dull and stated that they lost interest in the content. Some women suggested adding more visual material in the sessions. Furthermore, male heads of households were not included in the group sessions, and women found it difficult to follow the recommended changes in purchasing behavior as they were not the financial or primary decision-makers of the family. As such, women had difficulty adopting the purchase and use of items such as lead-free, unpolished turmeric and slatted lids to cover hot foods to prevent contamination from flies, and faced difficulty when trying to make safe and nutritious meals. The challenges and barriers to attendance and practicing behavioral recommendations are summarized in Table 3.

Modifications to intervention content. We made modifications to the delivery of the intervention to address these barriers. We asked women to bring family members who would watch over the children with the goal of decreasing distractions and increase attention during the sessions. In an attempt to increase attendance, create group accountability, and establish social support networks, we assigned names to individual groups, and encouraged women to identify and participate within these specific groups.

To promote attendance, we asked each participant, on a rotating basis, to serve as a group leader who would support the $\mathrm{CHW}$ in her organization of the sessions. As many women had difficulty in regularly attending sessions, the group leader was tasked with reminding women to attend sessions on time and communicating information covered, to women who were unable to attend the sessions.

To make the material more engaging, we implemented a storytelling format. Content on maternal mental health used a story-based format with a sequence of drawings portraying two mothers in opposite scenarios (one demonstrating healthy family practices and the other unhealthy).

To promote the uptake of WASH behaviors, we developed and facilitated the adoption of a self-administered tool for household assessment and planning. This process allowed participating households to identify areas for improvement and track any progress toward their personally identified goals for improving WASH infrastructure and behaviors within their household.

To incorporate household decision-makers into group discussions, we conducted two sessions involving male household decision-makers. In these male sessions, the roles and responsibilities of a household decision-maker were discussed. Behavioral recommendations including purchasing lead-free household products, prioritizing purchases of food from diverse food groups, and WASH hardware were described to male household members so that they could better play a supportive role to female household members.

Second qualitative assessment. We evaluated each modification to intervention delivery implemented after the first qualitative assessment.

Incorporating storytelling format within session contents. Mothers reported enjoying sessions more and paying more attention with the added storytelling format. Women could recall the different names of the characters from the stories and the behavioral recommendations described in the story. This better resonated with the women regarding topics of maternal depression and better captured their attention, especially during the longer sessions. One female participant in the group arm, high attendance group, during a focus group discussion said,

\begin{abstract}
After learning from the Amena ma character in our Thinking Healthy storytelling session, I take better care of my own health and my child's health. Also, I have learned that taking rest, getting proper sleep, and having a healthy diet can improve my health condition. I now have a better diet.
\end{abstract}

Bringing family members to assist with childcare. Female participants had suggested bringing a household member to care for their children, reduce distractions, and increase the overall operation in hopes that this would facilitate interactions among mothers and $\mathrm{CHWs}$, increase concentration among mothers, and decrease overall distractions within the session. When trying to implement this in the second phase, however, we found that many women did not have family members who were available to assist them during the entirety of the sessions. Consequently, this option was not realistic for many women. However, for those who did have family members available to attend and as exemplified during focus group discussions, mothers with high attendance from the group arm said that bringing family members to assist them in caring for their children during sessions was highly useful for them to concentrate in the session.

Incorporating household decision-makers into group discussions. Sessions involving male household decisionmakers were well attended, acceptable to group members, and may lead to improved adherence to behavioral recommendations.

All 27 participants from focus group discussions suggested that their husbands should attend the sessions and informed them of the sessions' content, time, frequency, and duration. Mothers hoped that the sessions would be beneficial to fathers with young children as they pertained directly to the 
child's health, hygiene, and diet. Some mothers reported that after attending the session, the husbands discussed with them what they learned and could understand the importance of their participation in the group sessions. Some mothers reported that male sessions helped the males understand the benefits of interventions which made it easier for them to attend the sessions. We conducted four in-depth interviews with fathers or grandfathers. Three stated that they did not face any difficulties in attending the meetings when they were conducted in the evenings.

Sessions with male participants were well received. All male in-depth interview participants (four) were satisfied with the content, behavior change communication materials used, and delivery method. They reported that the meeting was conducted well and deemed the $\mathrm{CHW}$ as an expert in explaining each of the interventions. As they were the primary decisionmakers in the households, the male sessions served to build support among the men for women's attendance at these sessions. The male sessions further helped women attend group sessions for reasons indicated in Table 3.

The participation of male household members helped families enact the behavioral recommendations such as selective purchasing of lead-free household products, diversified food for family, and WASH hardware. Some men who had attended the sessions reported changing their behavior in ways that may support the mental health of the mothers. A few mothers noted that their husbands now helped them in household chores at home. One 33-year-old male participant in the group arm in an in-depth interview said,

Previously I did not think like that; even I did not help my wife. For example, before, I would chat with my friends or neighbors during my free time, but now I stay at home and cooperate with my wife in her work and take care of my child. They are becoming happy and satisfied and even I feel better as well.

Another female participant in the combined arm stated in an in-depth interview that husbands learned material from the sessions.

Yes, my husband participated in one male session. The CHW told him about the meeting and invited him. Now my husband understands many things.

Incorporating household environmental assessment and planning. The household environmental assessment and planning tool was feasible to implement by $\mathrm{CHWs}$ and was accepted by the participants. This strategy included posters with illustrations and a pictorial booklet for self-administered household environmental assessment and progress-tracking by participants. A household environmental assessment and planning process was used to promote practicing WASH behavioral recommendations. In this process, the participants assessed their home environment and planned to increase adoption of WASH behaviors, and improved WASH infrastructure. At a later stage, they tracked their progress using the pictorial book. In the follow-up sessions, CHWs asked participants to recall what they had learned in the previous WASH session, and if necessary, participants discussed the problem(s) they were facing in implementing the improvement plan. From the focus group discussions with mothers, we identified that some WASH practices were followed. Some families $(n=6)$ used soapy water for handwashing by refilling a bottle with detergent, and some women $(n=4)$ reported washing their hands after cleaning a child's anus and disposing child feces. One 28-year-old female participant in combined arm with high-attendance focus group discussion stated,

We benefited from the soapy water bottle and we are continuing to use the bottle. We have poured detergent again and are using the bottle.

One female participant from the targeted household in the in-depth interview stated,

I liked the knowledge about the handwashing process and the key times. I did not have enough knowledge on that but after getting the information, we benefitted. Now we wash our hands with soapy water after using the toilet, cleaning a child's anus, and disposing of child feces.

However, some of WASH practices were not followed: no women reported cleaning hands with soap after disposing chicken feces, and many women $(n=15)$ still washed their hands with water only (no soap) after disposing of their child's feces. As a barrier to follow the behavioral recommendation, they mentioned their many household tasks. A 30-year-old mother from combined arm said,

I can't practice handwashing at all recommended times. I have to do a lot of household chores, I have to make fuel with cow dung, I have to feed my cattle, clean the cowshed. I wash my hands with soap after completing all my household chores.

Assigning a group leader within the session. Mothers who were assigned as a group leader were tasked with assisting $\mathrm{CHWs}$ and reminding mothers to attend group sessions and discussing the session topics with the absent mother participants. Most women from both focus group discussions and in-depth interviews could correctly identify the tasks of a group leader. One participant in the combined arm (low attendance group) expressed a positive opinion of the leader role and stated in the in-depth interview,

My sister in law was chosen to be a group leader. She was told to explain the topics to others who were not present or did not understand the topic very well. I don't think she considers it a burden. If I were chosen as a leader, I would also do the same.

Most of the women, however, faced difficulty in adopting a leader role because of cultural expectations of mobility. One participant in the group arm, high-attendance group, during a focus group discussion said,

We are daughters-in-law of this village; we are not permitted to go to other's houses without invitation. Even our in-laws will not permit us to roam around the village. So, it is difficult for us to become a group leader and perform the expected duties. 
Some refused the position or did not want to be chosen as they thought the tasks were burdensome. Those who declined the role also were not interested in suggesting an alternative group leader among the participants.

\section{DISCUSSION}

The key findings from our study are as follows: 1) adding a storytelling component to the group session increased interest and engagement of the mothers, 2) engaging other members of the household in intervention sessions improved family member understanding of the benefits of attending sessions and allowed for continued attendance, 3) including male sessions increased engagement of male household figures and helped the household decision-makers understand the necessity of the intervention, 4) the household environmental assessment and planning was feasible to implement and promoted some WASH behaviors, and 5) the group leader role was not successful as mothers often faced restricted mobility moving from household to household to carry out their role.

The storytelling component made the intervention more engaging and memorable to participants. Incorporation of storytelling into group sessions has some evidence of success, ${ }^{41,43}$ with the primary focus of engaging young children as the target group. One other study used a storytelling component in their integrated intervention in rural Bangladesh and found it effective when combined with flipcharts, cue cards, reenactments, and video to cover all behavioral objectives within the target households ${ }^{44}$; however, that study aimed to engage all household members, rather than individual mothers only. A third study applied a storytelling format that incorporated maternal, newborn, and child health activities to improve adoption of new behaviors. ${ }^{45}$ The successful development and implementation of story-based intervention delivery was an important achievement in our study. The challenges stemmed from attention and information retention during group sessions created some barriers in the feasibility and uptake of interventions at home, particularly due to the relatively long sessions that were too content-heavy for participants to absorb.

Children created a noisy and distracting environment for mothers, which undermined their ability to pay attention during session delivery. When family members accompanied mothers to intervention sessions ${ }^{46}$ to take care of their children, mothers were able to fully devote their attention to the group session. However, many participants did not have family members available to accompany them to sessions.

The CHWs were able to recruit and engage male household members for male-only sessions. A study conducted in Tanzania demonstrated that an antenatal group educational program focusing on engaging the male head of households increased knowledge and family support to the pregnant women. ${ }^{24,47}$ In our study, males who were previously not interested in sessions began to understand how it pertained to the long-term physical and cognitive health of their children. If male sessions were conducted earlier in the implementation time line, the men might have been more helpful in encouraging their wives to attend the sessions, which may have resulted in increased attendance rates during the study. Other studies have demonstrated the benefits of involving fathers in early childhood development. Particularly, fathers involved in child stimulation practices can provide important opportunities to buffer against high-risk, inadequate learning environments. ${ }^{48-50}$ Timing of when to incorporate male participation should be considered to successfully include males in the intervention. A study in Tanzania demonstrated that men often had no difficulties attending sessions, even when busy with their day-long agricultural work during harvesting season, as long as the meetings were conducted in the evening. ${ }^{51}$ In that study, an official letter was provided to the male partners to join antenatal care sessions and voluntary counseling sessions. ${ }^{51}$ In our study, the Bengali months of Ashar and Srabon (monsoon) were the most feasible period for men to attend meetings at any time of day as they had less work-related commitments during the harvesting season. However, holding sessions during the evening time was highly preferred by the few male participants we interviewed.

Women normally lack access to improved sanitation and often lack the decision-making power ${ }^{52}$ and financial means in the home to adopt sanitation technologies and strategies and sustain implementation of these integrated packages. In a study with a similar hygiene environment to ours, women with lower education from low-income communities lacked the decision-making power and self-efficacy to improve their water, sanitation, and health environment. ${ }^{53}$ In our study, we saw that when males were included in the sessions, there was greater acceptability of mothers attending because of the men's newfound understanding of the benefits of interventions and greater wiliness to invest in sanitation technologies within the household.

The household environmental assessment and planning component improved sanitation practices among participants due to its interactive nature over the previous conventional flipcharts and pictorial cards as it was visual and collaborative. Within our study, this added component provided a novel way to recognize and perceive good WASH practices after handling child and chicken feces. Previous home-based environmental education interventions like this have increased knowledge of the environmental hazards in the household and led to reduced exposures. ${ }^{54}$ Self-administered, group-based implementation directly by the study participants facilitated by $\mathrm{CHWs}$ can enable future implementation of home environmental interventions at scale.

Mothers in each group with young children were assigned a leader role, requiring them to move from house to house to carry out their role. Mobilizing women through a similar leader role had proved successful as it increased their participation and further improved their status in the communities. ${ }^{28}$ Our findings, in contrast, demonstrated that the role of family, social networks, and norms established by the patriarchal society negatively influenced the acceptability and feasibility of a female leader. Mothers with young children sometimes faced opposition from their families and were not allowed to go from household to household and execute their tasks. As a result, women rarely hold these kinds of leadership roles in rural areas, ${ }^{55}$ especially without a suitable incentive, because of the proscriptive norm that women remain in the home and refrain from engaging in outside activities. In the absence of support and approval from family members, it proved difficult for women to continue a leadership position, especially in settings like rural Bangladesh, where women 
are expected to remain under the rule of their fathers, husbands, and sons. ${ }^{55}$

In conclusion, it was acceptable and feasible for mothers with young children and pregnant women to attend the revised integrated group-based RINEW intervention and feasible to adopt the behavioral recommendations. Key revisions implemented in the study to improve acceptability included bringing family members for childcare assistance, adding a storytelling format in session contents, implementing a self-administered household environmental assessment including group discussions with household decision-makers, and assigning a group leader within participants for the sessions. Our group-based intervention shows promise for implementation at scale. This work shows that group sessions can be modified so that mothers are more willing and able to attend. Videos and other sources of visual stimulation should be used to make group meetings more interesting and meaningful. Future research developing and evaluating media-based messaging as a complement to group sessions or house to house visits might be an important strategy as it is more scalable than inperson visits. In addition, the duration of sessions should be modified to last 1 hour or less, as time played a large role in determining whether the participants were able to participate in the entirety of the session.

Received August 10, 2020. Accepted for publication November 24, 2020.

Published online January 18, 2021.

Acknowledgments: We are grateful to icddr,b's Environmental Interventions Unit. The icddr,b acknowledges with gratitude the study participants, the dedicated field team, and the communities. icddr,b is thankful to the governments of Bangladesh, Canada, Sweden, and the United Kingdom for providing core/unrestricted support.

Financial support: This research protocol/activity/study was funded by Gates Foundation Protocol number: PR-16037.

Authors' addresses: Farzana Yeasmin, Tania Jahir, Jyoti B. Das, Mohammad R. Amin, Md K. Hossain, Tarique Md. Nurul Huda, Fahmida Akter, Abul Kashem Shoab, Fahmida Tofail, Jesmin Sultana, and Mahbubur Rahman, International Center for Diarrheal Diseases Research, Bangladesh, Dhaka, Bangladesh, E-mails: fyeasmin@icddrb.org, tania.jahir@icddrb.org, jyoti.das@icddrb.org, ruhul.amin@icddrb.org, khobair.hossain@icddrb.org, tarique.huda@icddrb.org, fahmida.akter@ icddrb.org, akmshoab@icddrb.org, ftofail@icddrb.org, jesmin.sultana@ icddrb.org, and mahbubr@icddrb.org. Peter J. Winch, Sharon T. Hwang, and Elli Leontsini, Johns Hopkins Bloomberg School of Public Health University, Baltimore, MD, E-mails: pwinch@jhu.edu, sharon.hwang@ jhu.edu, and eleontsi@jhu.edu. Malay K. Mridha, James P. Grant School of Public Health, Dhaka, Bangladesh, E-mail: malay.mridha@bracu.ac.bd. Helen Pitchik and Lia C. H. Fernald, University of California, Berkeley, Berkeley, CA, E-mails: fernald@berkeley.edu and hpitchik@berkeley.edu. Stephen P. Luby, Stanford University, Stanford, CA, E-mail: sluby@ stanford.edu.

This is an open-access article distributed under the terms of the Creative Commons Attribution (CC-BY) License, which permits unrestricted use, distribution, and reproduction in any medium, provided the original author and source are credited.

\section{REFERENCES}

1. Lu C, Black MM, Richter LM, 2016. Risk of poor development in young children in low-income and middle-income countries: an estimation and analysis at the global, regional, and country level. Lancet Glob Health 4: e916-e922.

2. Blencowe H, Krasevec J, de Onis M, Black RE, An X, Stevens GA, Borghi E, Hayashi C, Estevez D, Cegolon L, 2019. National, regional, and worldwide estimates of low birthweight in 2015, with trends from 2000: a systematic analysis. Lancet Glob Health 7: e849-e860.

3. Hawkes C, Fanzo J, 2017. Nourishing the SDGs: Global Nutrition Report 2017. Bristol, UK: UNICEF.

4. Wiemeyer GM, Pérez MA, Bianchini LT, Sampietro L, Bravo GF, Jácome NL, Astore V, Lambertucci SA, 2017. Repeated conservation threats across the Americas: high levels of blood and bone lead in the Andean Condor widen the problem to a continental scale. Environ Pollut 220: 672-679.

5. Forsyth JE, Weaver KL, Maher K, Islam MS, Raqib R, Rahman M, Fendorf S, Luby SP, 2019. Sources of blood lead exposure in rural Bangladesh. Environ Sci Technol 53: 11429-11436.

6. Cuartas J, Jeong J, Rey-Guerra C, McCoy DC, Yoshikawa H, 2020. Maternal, paternal, and other caregivers' stimulation in low-and-middle-income countries. PLos One 15: e0236107.

7. Britto PR et al.; Early Childhood Development Interventions Review Group, for the Lancet Early Childhood Development Series Steering Committee, 2017. Nurturing care: promoting early childhood development. Lancet 389: 91-102.

8. Aboud FE, Yousafzai AK, 2015. Global health and development in early childhood. Annu Rev Psychol 66: 433-457.

9. Smuts CM, Matsungo TM, Malan L, Kruger HS, Rothman M, Kvalsvig JD, Covic N, Joosten K, Osendarp SJ, Bruins MJ, 2019. Effect of small-quantity lipid-based nutrient supplements on growth, psychomotor development, iron status, and morbidity among 6-to 12-mo-old infants in South Africa: a randomized controlled trial. Am J Clin Nutr 109: 55-68.

10. Oh C, Keats EC, Bhutta ZA, 2020. Vitamin and mineral supplementation during pregnancy on maternal, birth, child health and development outcomes in low-and middle-income countries: a systematic review and meta-analysis. Nutrients 12: 491.

11. Chang S, Zeng L, Brouwer ID, Kok FJ, Yan H, 2013. Effect of iron deficiency anemia in pregnancy on child mental development in rural China. Pediatrics 131: e755-e763.

12. Frongillo EA, Nguyen PH, Saha KK, Sanghvi T, Afsana K, Haque R, Baker J, Ruel MT, Rawat R, Menon P, 2017. Large-scale behavior-change initiative for infant and young child feeding advanced language and motor development in a clusterrandomized program evaluation in Bangladesh. Nutr $J$ 147: 256-263.

13. Lassi ZS, Rind F, Irfan O, Hadi R, Das JK, Bhutta ZA, 2020. Impact of infant and young child feeding (iycf) nutrition interventions on breastfeeding practices, growth and mortality in low-and middle-income countries: systematic review. Nutrients 12:722.

14. Gelaye B, Rondon MB, Araya R, Williams MA, 2016. Epidemiology of maternal depression, risk factors, and child outcomes in lowincome and middle- income countries. Lancet Psychiat 3: 973-982.

15. Grenier L, Suhowatsky S, Kabue MM, Noguchi LM, Mohan D, Karnad SR, Onguti B, Omanga E, Gichangi A, Wambua J, 2019. Impact of group antenatal care (G-ANC) versus individual antenatal care (ANC) on quality of care, ANC attendance and facility-based delivery: a pragmatic cluster-randomized controlled trial in Kenya and Nigeria. PLoS One 14: e0222177.

16. Chimbwete-Phiri R, Schnurr S, 2017. Negotiating knowledge and creating solidarity: humour in 614 antenatal counselling sessions at a rural hospital in Malawi. ScienceDirect 197: 68-82.

17. Mihanda RB, Likofata JR, Lusi GJ, 2013. Contribution of safe motherhood solidarity groups in using and accessing maternity services during a period of armed conflict. J Sci Res 5: 1085-1091.

18. Aboud FE, Singla DR, Nahil MI, Borisova I, 2013. Effectiveness of a parenting program in Bangladesh to address early childhood health, growth and development. Soc Sci Res 97: 250-258.

19. Dumas JE, Nissley-Tsiopinis J, Moreland AD, 2007. From intent to enrollment, attendance, and participation in preventive parenting groups. J Child Fam Stud 16: 1-26.

20. Paquin JD, Miles JR, Kivlighan DM, 2011. Predicting group attendance using in-session behaviors. Small Group Res 42: 177-198.

21. Shenderovich $Y$, Eisner $M$, Cluver L, Doubt J, Berezin $M$, Majokweni S, Murray AL, 2018. What affects attendance and engagement in a parenting program in South Africa? Prev Sci 19: 977-986. 
22. Gross D, Julion W, Fogg L, 2001. What motivates participation and dropout among low-income urban families of color in a prevention intervention? Fam Relat 50: 246-254.

23. Divan G, Hamdani SU, Vajartkar V, Minhas A, Taylor C, Aldred C, Leadbitter K, Rahman A, Green J, Patel V, 2015. Adapting an evidence-based intervention for autism spectrum disorder for scaling up in resource-constrained settings: the development of the PASS intervention in south Asia. Glob Health Action 8: 27278.

24. Shimpuku Y, Madeni FE, Horiuchi S, Kubota K, Leshabari SC, 2018. Evaluation of a family- oriented antenatal group educational program in rural Tanzania: a pre-test/post-test study. Reprod Health 15: 117.

25. Akter $\mathrm{F}$ et al., 2020. Adaptation and integration of psychosocial stimulation, maternal mental health and nutritional interventions for pregnant and lactating women in rural Bangladesh. Int J Environ Res Public Health 17: E6233.

26. Britt MK, Mohamadou S, Ashley V, Mahamadou T, Fatma LD, Katie ML, Diego B, 2020. Feasibility and preliminary effectiveness of group antenatal care in Senegalese health posts: a pilot implementation trial. Health Policy Plan 35: 587-599.

27. Massey Z, Rising SS, Ickovics J, 2006. CenteringPregnancy group prenatal care: promoting relationship-centered care. J Obstet Gynecol Neonatal Nurs 35: 286-294.

28. Morrison J, Tamang S, Mesko N, Osrin D, Shrestha B, Manandhar M, Manandhar D, Standing H, Costello A, 2005. Women's health groups to improve perinatal care in rural Nepal. BMC Pregnancy Childbirth 5: 6.

29. Roberton T, Applegate J, Lefevre AE, Mosha I, Cooper CM, Silverman M, Feldhaus I, Chebet JJ, Mpembeni R, Semu H, 2015. Initial experiences and innovations in supervising community health workers for maternal, newborn, and child health in Morogoro region, Tanzania. Hum Resour Health 13: 19.

30. Biswas S, Bose K, Mukhopadhyay A, Bhadra M, 2010. Mid-upper arm circumference based undernutrition among Bengalee children of Chapra, West Bengal, India. Iran J Pediat 20: 63-68.

31. Briend A, Maire B, Fontaine O, Garenne M, 2012. Mid-upper arm circumference and weight for height to identify high risk malnourished under-five children. Matern Child Nutr 8: 130-133.

32. Walker SP, Chang SM, Smith JA, Baker-Henningham H, 2018. The reach up early ChildhoodParenting program: origins, content, and implementation. Ann Ny Acad Sci 38: 37-43.

33. Unicomb L et al., 2018. WASH benefits Bangladesh trial: management structure for achieving high coverage in an efficacy trial. Trials 19: 359.

34. Ashraf S, Nizame FA, Islam M, Dutta NC, Yeasmin D, Akhter S, Abedin J, Winch PJ, Ram PK, Unicomb L, 2017. Nonrandomized trial of feasibility and acceptability of strategies for promotion of soapy water as a handwashing agent in rural Bangladesh. Am J Trop Med Hyg 96: 421-429.

35. Hulland KR, Leontsini E, Dreibelbis R, Unicomb L, Afroz A, Dutta NC, NizameFA, Luby SP, Ram PK, Winch PJ, 2013. Designing a handwashing station for infrastructure-restricted communities in Bangladesh using the integrated behavioural model for water, sanitation and hygiene interventions (IBM-WASH). BMC Public Health 13: 877 .

36. WHO, 2015. Thinking Healthy: A Manual for Psychosocial Management of Perinatal Depression, WHO Generic Field-Trial Version 1.0, 2015. Geneva, Switzerland: World Health Organization.

37. Dreibelbis R, Winch PJ, Leontsini E, Hulland KR, Ram PK, Unicomb L, Luby SP, 2013. The integrated behavioural model for water, sanitation, and hygiene: a systematic review of behavioural models and a framework for designing and evaluating behaviour change interventions in infrastructurerestricted settings. BMC Public Health 13: 1015.

38. Greenspan JA, McMahon SA, Chebet JJ, Mpunga M, Urassa DP, Winch PJ, 2013. Sources of community health worker motivation: a qualitative study in Morogoro Region, Tanzania. Hum Resour Health 11: 52.
39. Rahman SM et al., 2010. Factors affecting recruitment and retention of community health workers in a newborn care intervention in Bangladesh. Hum Resour Health 8: 12.

40. Krefting L, 1991. Rigor in qualitative research: the assessment of trustworthiness. Am J Occup Ther 45: 214-222.

41. McMahon SA, Winch PJ, Caruso BA, Obure AF, Ogutu EA, Ochari $I A$, Rheingans $R D, 2011$. 'The girl with her period is the one to hang her head' reflections on menstrual management among schoolgirls in rural Kenya. BMC Int Health Hum Rights 11: 7.

42. Sutton J, Austin Z, 2015. Qualitative research: data collection, analysis, and management. Can J Hosp Pharm 68: 226-231.

43. Winch PJ, Alam MA, Akther A, Afroz D, Ali NA, Ellis AA, Baqui AH, Darmstadt GL, El Arifeen S, Seraji MHR, 2005. Local understandings of vulnerability and protection during the neonatal period in Sylhet district, Bangladesh: a qualitative study. Lancet 366: 478-485.

44. Wagner JT, Samuelsson IP, 2019. WASH from the START: water, sanitation and hygiene education in preschool. Int J Early Child 51: 5-21.

45. Luby SP, Rahman M, Arnold BF, Unicomb L, Ashraf S, Winch PJ, Stewart CP, Begum F, Hussain F, Benjamin-Chung J, 2018. Effects of water quality, sanitation, handwashing, and nutritional interventions on diarrhoea and child growth in rural Bangladesh: a cluster randomised controlled trial. Lancet Glob Health 6: e302-e315.

46. Cooper CM, Ahmed S, Winch PJ, Pfitzer A, McKaig C, Baqui AH, 2014. Findings from the use of a narrative story and leaflet to influence shifts along the behavior change continuum toward postpartum contraceptive uptake in Sylhet district, Bangladesh. Patient Educ Couns 97: 376-382.

47. Thapa DK, Niehof A, 2013. Women's autonomy and husbands' involvement in maternal health care in Nepal. Soc Sci Med 93: $1-10$.

48. Singla DR, Kumbakumba E, Aboud FE, 2015. Effects of a parenting intervention to address maternal psychological wellbeing and child development and growth in rural Uganda: a community-based, cluster-randomised trial. Lancet Glob Health 3: e458-e469.

49. Jeong J, McCoy DC, Fink G, 2017. Pathways between paternal and maternal education, caregivers' support for learning, and early child development in 44 low-and middle-income countries. Early Child Res Q 41: 136-148.

50. Jeong J, McCoy DC, Yousafzai AK, Salhi C, Fink G, 2016. Paternal stimulation and early child development in low-and middleincome countries. Pediatrics 138: e20161357.

51. Betancourt TS, Jensen SK, Barnhart DA, Brennan RT, Murray SM, Yousafzai AK, Farrar J, Godfroid K, Bazubagira SM, Rawlings LB, 2020. Promoting parent-child relationships and preventing violence via home-visiting: a pre-post cluster randomised trial among Rwandan families linked to social protection programmes. BMC Public Health 20: 621.

52. Jefferys LF, Nchimbi P, Mbezi P, Sewangi J, Theuring S, 2015. Official invitation letters to promote male partner attendance and couple voluntary HIV counselling and testing in antenatal care: an implementation study in Mbeya region, Tanzania. Reprod Health 12: 95.

53. Rajaraman D, Varadharajan KS, Greenland K, Curtis V, Kumar R, Schmidt WP, Aunger R, Biran A, 2014. Implementing effective hygiene promotion: lessons from the process evaluation of an intervention to promote handwashing with soap in rural India. BMC Public Health 14: 1179.

54. Mankikar D, Campbell C, Greenberg R, 2016. Evaluation of a home-based environmental and educational intervention to improve health in vulnerable households: southeastern Pennsylvania lead and healthy homes program. Int $J$ Environ Res Public Health 13: 900.

55. Sherman A, 2000. Women managing/managing women: the marginalization of female leadership in rural school settings. Educ Manag Adm Leadersh 28: 133-143. 